\title{
A novel vector for positive selection of inserts harboring an open reading frame by translational coupling
}

\author{
Sumiko Ohashi-Kunihiro', ${ }^{1,}$, Masafumi Yohda², Haruhiko Masaki ${ }^{3}$, and \\ Masayuki Machida ${ }^{1}$ \\ ${ }^{1}$ Research Institute for Cell Engineering, National Institute of Advanced Industrial Science \\ and Technology (AIST), Higashi, Tsukuba, Ibaraki, 2Department of Biotechnology and \\ Life Science, Tokyo University of Agriculture and Technology, and ${ }^{3}$ Department of \\ Biotechnology, Graduate School of Agricultural and Life Sciences, The University of \\ Tokyo, Tokyo, Japan
}

BioTechniques 43:751-754 (December 2007)

doi 10.2144/000112629

We have developed a novel vector, pTCS, as a tool for efficient selection of open reading frame (ORF)-containing inserts. In pTCS clones containing an insert with an ORF, a downstream marker gene (immE3, conferring resistance to colicin) is activated via translational coupling with the insert, and transformed cells can then be selected by exposure to colicin E3. Our method differs from previous methods in that the marker gene is activated without protein fusion, and that selection occurs irrespective of the reading frame of the insert.

Comprehensive analysis of gene function requires construction and screening of expression libraries containing DNA fragments; however, during construction of the libraries, control of the reading frame of the inserts is important for screening efficiency. To permit enrichment for authentic translation products, several open reading frame (ORF) selection vectors, which involve protein fusion between DNA inserts and selectable marker genes, have been developed $(1,2)$. Fusion of a foreign protein to a reporter protein often causes significant loss of activity or inactivation of the protein of interest $(3,4)$. In addition, the pKE- 1 vector selects positively no more than two out of the three downstream reading frames of cDNAs, utilizing the phenomenon whereby translation can reinitiate without a ribosome binding site if an AUG codon exists within a few nucleotides of the stop codon of the preceding translated sequence (5).

In an effort to overcome the above limitations (protein fusion and reading frames) and design a more effective method of ORF-specific screening, we developed a novel vector, pTCS (GenBank accession no. AB353144), which functions via "translational coupling" of in-frame inserts and a selectable marker gene. On plasmid ColE3, colicin E3 (colE3), which kills susceptible Escherichia coli cells (6) and the immunity E3 (immE3), which neutralizes the colicin E3, face the same direction with an intergenic space of nine nucleotides (7), and their expression can be "translationally coupled" (8). In the present study, we used immE3 as a selectable marker gene and selected cells using colicin E3. The intention was that in-frame inserts would confer colicin E3 resistance upon the host by activating expression of $i m m E 3$, whereas the vector alone and inserts containing in-frame stop codon(s) would not (Figure 1).

To achieve this translational coupling of inserts and immE3, and to achieve efficient selection independent of the downstream reading frame of inserts, we designed a novel sequence, TGAATGAATGATG, which contains three termination codons (underlined) in different translational reading frames and an initiation codon (italicized) for immE3 overlapping with the third termination codon. The intergenic spaces for colicin and the immunity genes in strains of $E$. coli producing classes E2, E3, E8, E9, and $\mathrm{D}$ of colicin are similar, ranging from $-2 \mathrm{bp}$ (partially overlapping) to $9 \mathrm{bp}$. Therefore, for TGAATGAATGATG, we hypothesized that distances of $-1 \mathrm{bp}, 3 \mathrm{bp}$, and $7 \mathrm{bp}$, respectively, would permit translational coupling. In order to confirm that the sequence functions as a sensor for detecting the translational read-through of an insert, six different inserts containing the influenza virus hemagglutinin (HA) epitope sequence (33 bp) were prepared to yield six possible translation outcomes. Fragments containing the HA epitope sequence, the novel sequence, and immE3 were prepared from $\mathrm{pSH} 350$ (9) by PCR (Expand High-Fidelity PCR System; Roche Diagnostics, Basel, Switzerland), then cloned into pUC19

Table 1. Number of Colonies Obtained through Positive Selection

\begin{tabular}{|c|c|c|}
\hline \multirow{2}{*}{ Fragment } & \multicolumn{2}{|c|}{ Colicin } \\
\hline & + & - \\
\hline Avi-1 & $3 \times 10^{4}$ & $5 \times 10^{4}$ \\
\hline Avi-2 & $>10^{6}$ & $>10^{6}$ \\
\hline Avi-3 & $>10^{6}$ & $>10^{6}$ \\
\hline TAA-Avi1 & $9 \times 10^{2}$ & $6 \times 10^{5}$ \\
\hline TAA-Avi2 & $1 \times 10^{3}$ & $>10^{6}$ \\
\hline TAA-Avi3 & $3 \times 10^{2}$ & $2 \times 10^{4}$ \\
\hline \multicolumn{3}{|c|}{$\begin{array}{l}\text { Six DNA fragments, Avi-1, Avi-2, Avi-3, TAA-Avi1, TAA-Avi2, and TAA-Avi3, containing the synthetic strep- } \\
\text { tavidin-encoding gene were used as inserts. Avi series, which all had ORF-containing inserts, differed } \\
\text { from each other by one nucleotide in length at the } 3^{\prime} \text {-end of the synthetic streptavidin-encoding gene, } \\
\text { and TAA-Avi series all had termination codons at their } 5^{\prime} \text {-ends. These fragments were ligated at an ap- } \\
\text { proximately } 1: 1 \text { (vector:insert) molar ratio with pTCS vector (10-20 fmoles), and transformed into XLI-Blue } \\
\text { MRF }{ }^{\prime} \text { according to industrial protocol. A portion of the transformed cells (approximately } 50 \mu \mathrm{ll} \text { ) suspended } \\
\text { in SOC medium were cultivated in } 400 \mu \mathrm{LB} \text { L broth containing } 1 \mathrm{mM} \text { IPTG at } 37^{\circ} \mathrm{C} \text { for } 6 \mathrm{~h} \text {, divided in two, } \\
\text { and plated on the LB/ampicillin/IPTG plates with [colicin }(+) \text { ] or without [colicin }(-) \text { ] addition of } 10 \mu \mathrm{g} / \mathrm{mL} \\
\text { colicin E3, respectively. The numbers of colonies formed on each plate are indicated. }\end{array}$} \\
\hline
\end{tabular}




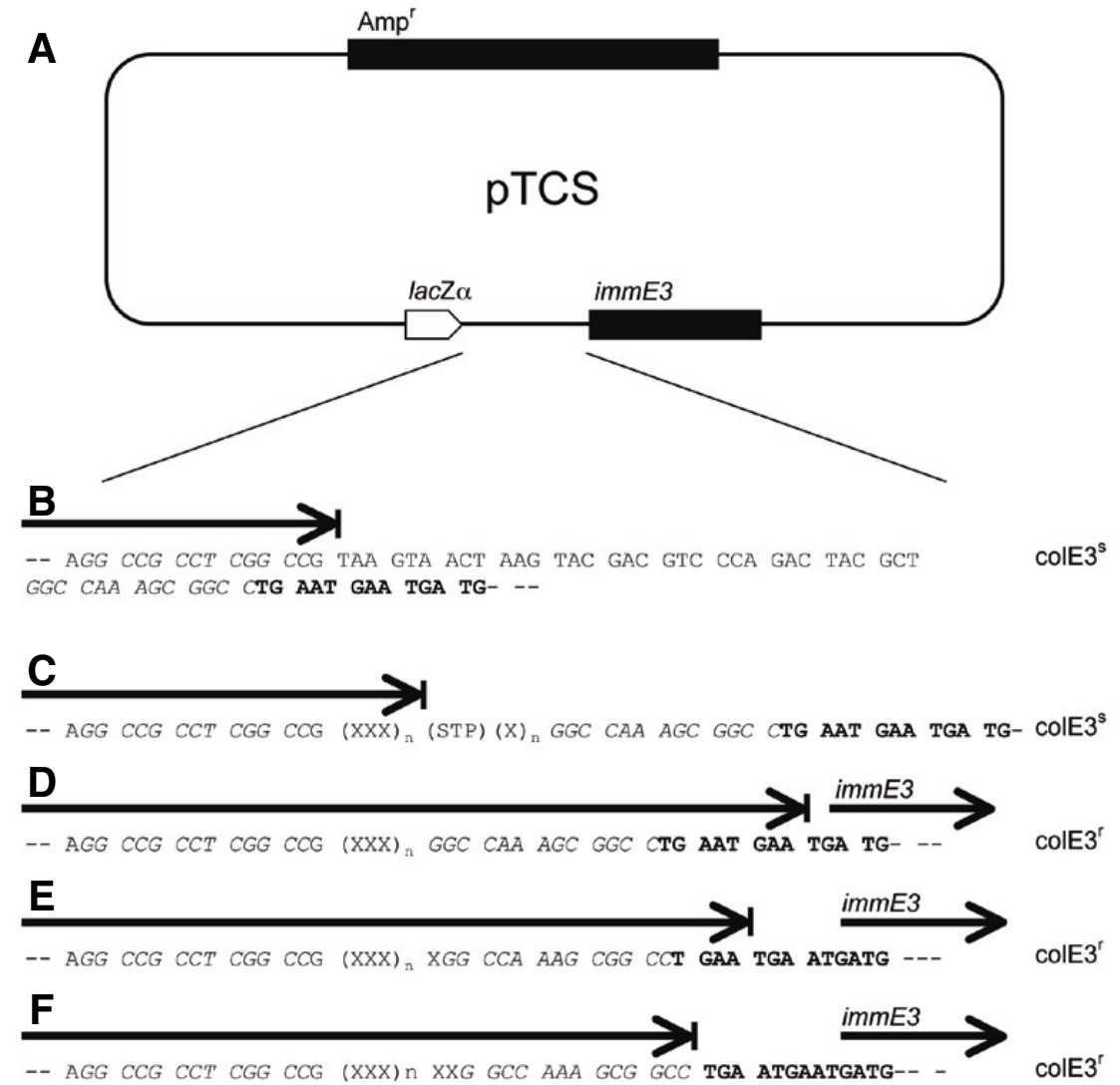

Figure 1. Design and function of the pTCS vector. (A) pTCS was derived from pUC19. It contains a short amino-terminal fragment of $L a c Z \alpha$ under the control of the inducible lac promoter; two SfiI sites generating sticky ends of different sequences from each other to allow directional cloning; a novel sequence, TGAATGAATGATG; and the immE3 coding region. (B) Sequence between the short amino-terminal fragment of $L a c Z \alpha$ and the immE3 ATG start codon. The two SfiI sites and the novel sequence are indicated in italic and bold letters, respectively. The arrow indicates the translation path, and nucleotide triplets show the reading frame. Escherichia coli cells containing pTCS alone are colicin E3-sensitive (colE3), because a stop codon between the two SfiI sites interrupts translation from the $L a c Z \alpha$ start codon and immE3 is not translated. (C-F) Inserts are represented by X's. (C) A fragment containing in-frame stop codon(s). (STP) indicates the stop codon(s). Transformants containing plasmids with this insert were also colicin E3-sensitive. Fragments with (D) a multiple of three, (E) a multiple of three plus one, (F) a multiple of three plus two in length. In these cases, immE3 is translated by complete translation of fragments up to the in-frame TGA stop codon of the novel sequence. Thus, the host cells are colicin E3-resistant (colE3r).

(Takara, Otsu, Shiga, Japan) for expression under the control of the lac promoter, and transformed into $E$. coli XLI-Blue MRF' (Stratagene, La Jolla, CA, USA) (see Supplementary Figure S1 available online at www. BioTechniques.com). HAimm-1, HAimm-2, and HAimm-3, which all had ORF-containing inserts, differed from each other by one nucleotide in length at the 3 -end of the HA epitope sequence. HAimm-t1, HAimm-t2, and HAimm-t 3 all had termination codons at their 5'-ends. Colicin E3 was prepared from W3110Str (ColE3-CA38) and purified using the method of Herschman and Helinski (10), except that $0.5 \mathrm{M}$ guanidine hydrochloride was used instead of $1 \mathrm{M} \mathrm{NaCl}$ for extracting E3 (11). Transformant sensitivity to colicin E3 was tested on plates containing 100 $\mu \mathrm{g}$ ampicillin/mL plus $200 \mu \mathrm{M}$ IPTG using the cross-streak test described by Takagaki et al. in Reference 12 . The HAimm-t1, HAimm-t2, and HAimm-t 3 transformants were all sensitive to colicin E3, whereas the HAimm-1, HAimm-2, and HAimm3 transformants were not. We therefore surmised that immE3 was translated only if translation of the insert was not terminated before the novel sequence was reached. Using this mechanism, other marker genes could be used instead of immE3, including other genes conferring drug tolerance, and genes with other functions. Given the above results, we then constructed the pTCS vector, which contained immE3 downstream of the novel sequence. Upstream of the novel sequence contained two SfiI sites (13), which had different sequences at the three-base cohesive ends to allow directional cloning with single enzyme digestion (Figure 1) (see Supplementary Figure S2).

In order to evaluate the efficiency of selection using the pTCS vector, six DNA fragments containing the synthetic streptavidin-encoding gene (387 bp) (14), each with an SfiI site at both ends, were prepared by PCR in the same manner as the HAimm fragments. The fragments were cloned into the SfiI site of the pTCS vector, and the vector was transformed into E. coli cells. After induction of the lac promoter using $1 \mathrm{mM} \mathrm{IPTG} \mathrm{(at} 37^{\circ} \mathrm{C}$ for approximately $6 \mathrm{~h}$ with shaking), colicin E3 protein was added at a final concentration of $10 \mu \mathrm{g} / \mathrm{mL}$ and the transformants were immediately plated onto LB plates containing 100 $\mu \mathrm{g}$ ampicillin/mL plus $200 \mu \mathrm{M}$ IPTG. Comparing colony formation of six transformed cells between those with and without colicin E3 protein, three transformed cells containing clones with an insert cloned in frame (Avi1, Avi2, and Avi3 fragments) formed similar number of colonies, respectively. In contrast, the colony numbers of three transformed cells containing clones with an insert cloned out of frame (TAA-Avi1, TAA-Avi2, and TAA-Avi3) were dramatically decreased upon addition of colicin E3 protein (Table 1). Sequencing of some clones obtained from these transformants revealed mutations that were probably introduced during PCR amplification or during cultivation, which allowed translational read-though. (see Supplementary Table S1). It is possible that the long duration of IPTG induction before selection $(6 \mathrm{~h})$ may have permitted significant growth of $E$. coli cells in the absence of selection pressure. Subsequent experiments showed that $2 \mathrm{~h}$ of IPTG induction and $3 \mu \mathrm{g} / \mathrm{mL}$ colicin E3 for subsequent growth were optimal (data not shown). 


\section{Benchmarks}

Expression of inserts introduced between the SfiI sites is under the control of the lac promoter and positive selection of transformants containing pTCS with ORF-containing DNA fragments is carried out in the presence of colicin E3. Introduction of fragments that contain the only one termination codon (TAA) in frame suppresses the expression of immE3. This process enables selection for DNA fragments derived from structural genes, including those prepared from cDNA and fragmented genomic DNA. However, inserts containing coding sequences with the native stop codon may be missed if there is too much distance between the native stop codon and the start of the immunity gene so as to prevent translational coupling. Except in such circumstances, notably, pTCS is able to activate the marker gene without protein fusion, and permits selection irrespective of the downstream reading frame of an insert. Use of the pTCS plasmid for efficient selection of any insert without an inframe stop codon, regardless of the downstream reading frame, is likely to be a useful technique in comprehensive gene function analysis.

\section{ACKNOWLEDGMENTS}

The research was supported by a grant from the New Energy and Industrial Technology Development Organization (NEDO), Japan.

\section{COMPETING INTERESTS STATEMENT}

The authors declare no competing interests.

\section{REFERENCES}

1. Daugelat, S. and W.R. Jacobs, Jr. 1999. The Mycobacterium tuberculosis recA intein can be used in an ORFTRAP to select for open reading frames. Protein Sci. 8:644-653.

2. Rombel, I.T., K.F. Sykes, S. Rayner, and S.A. Johnston. 2002. ORF-FINDER: a vector for high-throughput gene identification. Gene 282:33-41.

3. Ladner, R.C. 1996. Display and selection of proteins on genetic packages, p. 151-194. In B.K. Kay, J. Winter, and J. McCafferty (Eds.), Phage Display of Peptides and Proteins: A
Laboratory Manual. Academic Press, San Diego, CA.

4. Das, A. 1990. Optimization of starting materials, p. 93-146. In M.P. Deutscher (Ed.), Guide to Protein Purification. Academic Press, San Diego, CA.

5. Davis, C.A. and S. Benzer. 1997. Generation of cDNA expression libraries enriched for inframe sequences. Proc. Natl. Acad. Sci. USA 94:2128-2132.

6. Pugsley, A.P. and B. Oudega. 1987 . Methods for studying colicins and their plasmids, p. 105-161. In K.G. Hardy (Ed.), Plasmids: A Practical Approach. IRL Press, Oxford, UK.

7. Masaki, H. and T. Ohta. 1982. A plasmid region encoding the active fragment and the inhibitor protein of colicin E3-CA38. FEBS Lett. 149:129-132.

8. Lau, P.C., R.W. Rowsome, M. Zuker, and L.P. Visentin. 1984. Comparative nucleotide sequences encoding the immunity proteins and the carboxyl-terminal peptides of colicins E2 and E3. Nucleic Acids Res. 12:87338745 .

9. Toba, M., H. Masaki, and T. Ohta. 1986. Primary structures of the ColE2-P9 and ColE3-CA38 lysis genes. J. Biochem. (Tokyo) 99:591-596.

10. Herschman, H.R. and D.R. Helinski. 1967. Purification and characterization of colicin E2 and colicin E3. J. Biol. Chem. 242:53605368.

11. Masaki, H. and T. Ohta. 1985. Colicin E3 and its immunity genes. J. Mol. Biol. 182:217-227.

12. Takagaki, Y., K. Kunugita, and M. Matsuhashi. 1973. Evidence for the direct action of colicin $\mathrm{K}$ on aerobic ${ }^{32} \mathrm{P}_{\mathrm{i}}$ uptake in Escherichia coli in vivo and in vitro. J. Bacteriol. 113:42-50.

13. Christian, R.B., R.N. Zuckermann, J.M. Kerr, L. Wang, and B.A. Malcolm. 1992. Simplified methods for construction, assessment and rapid screening of peptide libraries in bacteriophage. J. Mol. Biol. 227:711-718.

14. Thompson, L.D. and P.C. Weber. 1993. Construction and expression of a synthetic streptavidin-encoding gene in Escherichia coli. Gene 136:243-246.

Received 28 May 2007; accepted 1 October 2007.

Address correspondence to Masayuki Machida, National Institute of Advanced Industrial Science and Technology (AIST), Central 6, 1-1, Higashi, Tsukuba, Ibaraki, 305-8566, Japan. e-mail: m.machida@aist.go.jp

To purchase reprints of this article, contact: Reprints@BioTechniques.com 